\title{
Functional Outcome in Patients with Chronic Plantar Fasciitis Treated with Plantar Fascia Stetching vs Tendoachilles Stretching Exercises
}

\section{Jha R K, Uprety S, Shah L L}

Department of orthopaedics, Institute of Medicine, TUTH, Kathmandu, Nepal

Correspondence Address: Suresh Uprety, Department of orthopaedics, Institute of Medicine, TUTH, Kathmandu, Nepal

\begin{abstract}
Introduction: Approximately ten percentage of patients with plantar fasciitis have development of persistent and often disabling symptoms. A poor response to treatment may be due, in part, to inappropriate and nonspecific stretching techniques. We hypothesized that patients with chronic plantar fasciitis who are managed with the structure-specific plantar fascia-stretching program for eight weeks have a better functional outcome than do patients managed with a standard Achilles tendon-stretching protocol.

Methods: sixty five patients who had chronic proximal plantar fasciitis for duration of at least ten months were randomized into one of two treatment groups. All patients received prefabricated soft insoles and a three-week course of naproxen. The patients received instructions for either a plantar fascia tissue-stretching program (Group A) or an Achilles tendon-stretching program (Group B). All patients completed the pain subscale of the Foot Function Index and a subjectrelevant outcome survey that incorporated generic and condition-specific outcome measures related to pain, function, and satisfaction with treatment outcome. The patients were reevaluated after eight weeks.

Results: sixty one patients returned for follow-up evaluation (78 total feet). Covariates for baseline measures revealed no significant differences between the groups. The pain subscale scores of the Foot Function Index showed significantly better results for the patients managed with the plantar fascia stretching program with respect to item 1 (worst pain; $p=0.002$ ), item 2 (first steps in the morning; $\mathrm{p}=0.018)$ and combined items 1 to $7(\mathrm{p}=0.033)$.

Analysis of the response rates to the outcome measures also revealed significant differences with respect to pain, activity limitations, and patient satisfaction, with greater improvement seen in the group managed with the plantar fascia-stretching program.

Conclusions: A program of non-weight-bearing stretching exercises specific to the plantar fascia is superior to the standard program of weight-bearing Achilles tendon-stretching exercises for the treatment of symptoms of proximal plantar fasciitis. These findings provide an alternative option to the present standard of care in the non-operative treatment of patients with chronic, disabling plantar heel pain.
\end{abstract}

Key words: Plantar fasciitis, plantar fascia stretching, Tendoachilles stretching.

\section{Introduction}

Proximal plantar fasciitis is a common pathological condition in the adult, affecting the hindfoot, and can often be a challenge for clinicians to treat successfully. ${ }^{1,2}$ It occurs over a wide age range and is seen in both sedentary and athletic individuals. Although its precise cause remains 
unclear, the most common theory is repetitive partial tearing and chronic inflammation of the plantar fascia at its insertion on the medial tubercle of the calcaneus. ${ }^{3,4}$

A wide variety of management strategies have been developed to treat plantar fasciitis. Nonsurgical treatment is the mainstay of treating plantar fasciitis. ${ }^{5}$ Successful treatment of plantar fasciitis usually requires a combination of treatment modalities, rather than administering only one treatment at a time. ${ }^{6}$

The mechanical treatment should be considered a cornerstone of any plan of treatment. ${ }^{6,7}$, The aim of mechanical treatment modalities is to reduce the load and stress applied to inflamed plantar fascia during activity to a tolerable level. ${ }^{1,7}$

Although the majority of patients with plantar fasciitis have resolution of the symptoms within ten months, approximately $10 \%$ have development of persistent and often disabling symptoms. ${ }^{8}$ A poor response to treatment may be due, in part, to inappropriate and nonspecific stretching techniques or to improper recommendations for shoe inserts. Stretching protocols given to patients by physicians and physical therapists often emphasize stretching the Achilles tendon, but they do not specifically address the plantar fascia. ${ }^{8,9,13}$. The response of patients with chronic (ten months or more) disabling proximal plantar fasciitis to a treatment protocol emphasizing structure-specific plantar fascia-stretching combined with the use of a prefabricated, flexible orthotic device and a brief course of non-steroidal anti-inflammatory medications showed improvement in symptoms in response to the above treatment protocol.10 We hypothesize that Patients with chronic plantar fasciitis who are managed with a tissue-specific plantar fascia stretching protocol have a better functional outcome after eight weeks of treatment compared with that after a standard Achilles tendon-stretching protocol.

\section{Methods}

Between Nov 2008 to May 2010, sixty five patients (eighty three involved feet) who came to TUTH OPD with sign and symptoms of chronic plantar fasciitis for at least ten months were enrolled in this study. All patients complained of maximum pain upon palpation of the origin of the plantar fascia on the medial calcaneal tubercle, consistent with a diagnosis of proximal plantar fasciitis, with or without any previous non-operative treatments but had failed to respond to treatment. Patients were excluded if they had a history of systemic inflammatory disease, diabetes mellitus, prior heel surgery, or heel pain that is not consistent with proximal plantar fascitis, inability or unwillingness to discontinue current treatment modalities that are used for the purpose of plantar fasciitis, age of less than eighteen years, anatomical abnormality of foot and ankle and neurologic abnormality (changes of deep tendon reflexes, motor or sensory deficit). Written informed consent were taken from the patients who fell into the inclusion criteria of the study. Randomization of the treatment group was done by lottery method.

The study was approved by the Institutional Review Board of Institute of Medicine (IOM) and was conducted in the Orthopaedic department, Tribhuvan University teaching hospital, Maharajgunj, Kathmandu, Nepal.

\section{Procedure}

The patients initially completed a self-administered questionnaire that provided background information and a history profile of the heel pain. The background information included age, gender, height and weight, hours spent standing during the day, duration of symptoms, and types of prior treatments.

Patients who met the inclusion criteria for the study were then enrolled into either Group A (plantar fascia stretching exercise) or Group B (tendo-achilles stretching exercise). Patients in both groups were given prefabricated full-length soft insole and a three-week course of a non-steroidal antiinflammatory medication naproxen $250 \mathrm{mg}$ twice a day.

Patients who were randomized to treatment Group A received instructions in a plantar fascia tissue-stretching program as shown in figure 1 .

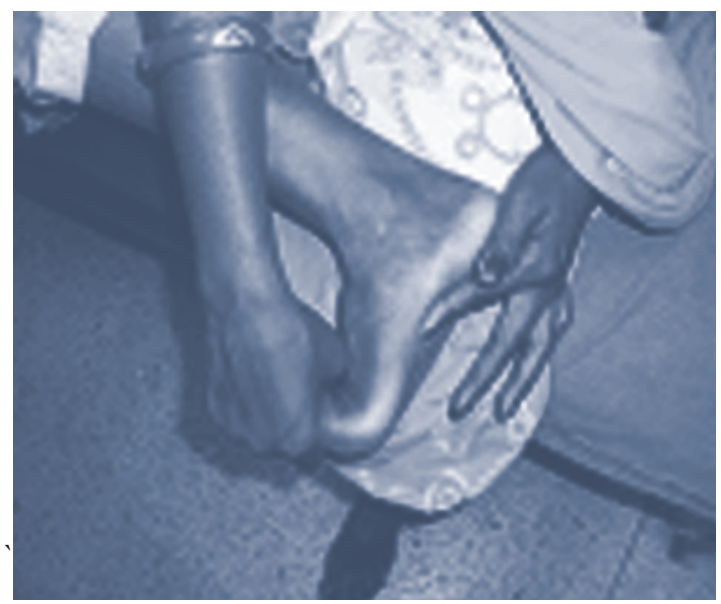

Figure 1 Plantar fascia-stretching exercise

The patient crossed the affected leg over the contralateral leg. While placing the fingers of same side of hand across the base of the toes, the patient pulled the toes back toward the shin until he or she felt a stretch in the arch or plantar fascia. The patient confirmed that the stretch was correct by palpating tension in the plantar fascia. 
Patients who were randomized into treatment Group B received instructions in an Achilles tendon-stretching program as shown in figure 2 .

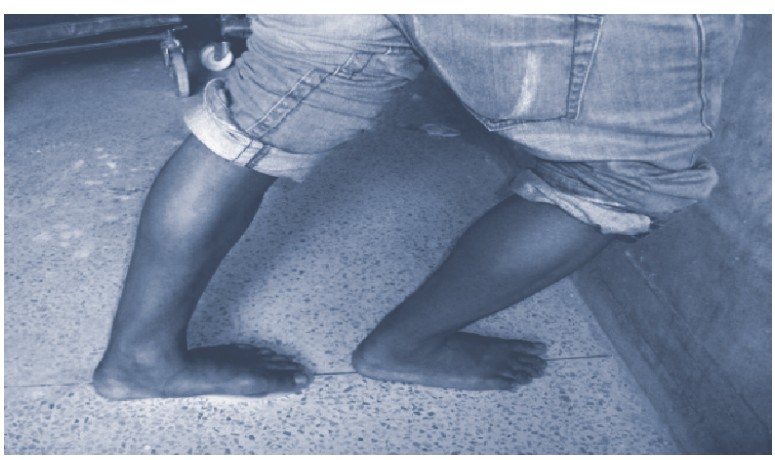

Figure 2 Achilles tendon-stretching exercise

The patient was instructed to place the shoe insert under the affected foot. He or she was then told to place the affected leg behind the contralateral leg, with the toes of the affected foot pointed toward the heel of the front foot, and to lean into the wall. The patient was then instructed to bend the front knee while keeping the back knee straight and the heel firmly on the floor.

Patients in both groups were instructed to hold each stretch for ten seconds (a count of ten) and to repeat it ten times. They were asked to perform the stretching program three times per day. For patients in Group A (plantar fasciastretching program), the first stretch was to be done before taking the first step in the morning. For patients in Group B (Achilles tendon stretching program), the first stretch was to be done immediately after getting out of bed in the morning. They were checked again to ensure that they were carrying out the exercises correctly. The patients were asked to discontinue any previous therapy that they were receiving for the heel pain. They were also encouraged not to change their regular shoe wear or activity level.

Before treatment, the patients completed the pain sub-scale of the Foot Function Index and a questionnaire related to activity level and function. At four weeks, the patients were asked to follow up to check for proper technique of exercises and any questions regarding the exercise protocols and to encourage continued participation. Patients returned at eight weeks for a follow-up examination and completion of the pain sub-scale of the Foot Function Index and a subjectrelevant outcome measures questionnaire that incorporated generic and condition-specific outcome measures related to pain, function, and satisfaction. The outcomes of the study were analyzed by stastician.

Questions from the pain subscale of the Foot Function
Index were used to generate the primary numeric outcome scores. The questions were scored from 0 (no pain) to 10 (worst pain imaginable), depending on the location marked by the patients on the visual analog scale. Only the first seven items were used to generate an overall score. The remaining two items on the pain subscale were related to orthotic use and were not relevant to all subjects. The sum of the scores on the first seven items was then expressed as a percentage of the maximum possible score, resulting in an overall percentage score that ranged from 0 (no pain on any question) to 100 (worst pain imaginable on all applicable questions).

The change in the overall pain score - that is, the score after eight weeks minus the baseline score-was used for subsequent analysis. Note that a negative change in the visual analog scale score signifies patient improvement. Additionally, as part of the study protocol, the changes in the numeric scores for the first two items on the pain subscale were selected a priori to be evaluated separately, as they were considered to represent the primary concerns articulated by patients with chronic heel pain.

\section{Statistical analysis}

Tests were conducted to assess the similarity of the groups on baseline measures. T tests were used for continuous data. Differences between the groups with respect to changes in the visual analog scale scores for the pain subscale of the Foot Function Index were analyzed with use of standard statistical procedures. Independent sample $t$ tests were used except in instances of violation of normal assumption, in which case the nonparametric equivalent was applied. An overall significance level was maintained at $\mathrm{p}<0.05$.

At the follow-up visit, patients were also asked to rate the change in the pain between the initial and follow-up visits as well as their perceptions of overall improvement since beginning the study. In order to simplify the interpretation of the analyses, the responses to the questions on the subject-relevant outcome measures were collapsed into dichotomized data indicating a positive response versus a non-response. Non-response represented little or no improvement. The association of treatment with response rates was analyzed. An overall significance level was maintained at $\mathrm{p}<0.05$.

\section{Results}

Of the sixty five patients with 83 involved feet found eligible for the study, four patients did not come for follow up. Out of four, there were two from each group with one enrolled in Group A had bilateral involvement. Out of total sixty one patient, there were thirty patients (37 feet) 
in plantar fascia stretching and thirty-one patients(41feet) in Tendo-achilles stretching group. The analysis of baseline measures for the two groups revealed that they were very similar with regard to age, body-mass index, and duration of hours standing per day. Covariates for baseline measures demonstrated no significant differences between the two groups as shown in table 1.

Table1: Baseline characteristics of the treatment group.

\begin{tabular}{lll} 
Measurement & Group A $(\mathbf{n}=\mathbf{3 0})$ & Group B (n= 31) \\
\hline Age $(\mathrm{yr}) \dagger$ & $41.87 \pm 8.07$ & $40.39 \pm 8.29$ \\
\hline Gender $(\mathrm{F} / \mathrm{M})$ & $22 / 8$ & $23 / 8$ \\
\hline Weight $(\mathrm{kg}) \dagger$ & $70.40 \mathrm{~kg} \pm 6.60$ & $71.26 \pm 7.08$ \\
\hline Body-mass index $(\mathrm{kg} / \mathrm{m} 2) \dagger$ & $29.08 \pm 1.93$ & $29.05 \pm 1.89$ \\
\hline Side of involvement $(\mathrm{U} / \mathrm{B})^{*}$ & $23 / 7$ & $21 / 10$ \\
\hline Involved side(Rt/Lt) & $20 / 16$ & $17 / 25$ \\
\hline No. of hours standing $\dagger$ & $6.87 \pm 1.852$ & $6.58 \pm 1.803$ \\
\hline Duration of symptoms & & \\
\hline $10-12$ mo & 16 & 11 \\
\hline $13-18$ mo & 13 & 18 \\
\hline $19-24$ mo & 5 & 10 \\
\hline $25-36$ mo & 1 & 3 \\
$>36$ mo & 1 & 0 \\
\hline
\end{tabular}

Group A was managed with a plantar fascia-stretching program, and Group B was managed with an Achilles tendon-stretching program

$\dagger$ The values are given as the mean, with the standard deviation.

* U/B, unilateral and bilateral involvement of feet

- involved right and left leg of patients

\section{Discussion}

Proximal plantar fasciitis is a common pathological condition in the adult, affecting the hindfoot, and can often be a challenge for clinicians to treat successfully. ${ }^{1,2}$ Although the majority of patients with plantar fasciitis have resolution of the symptoms within ten months, approximately $10 \%$ have development of persistent and often disabling symptoms. ${ }^{8}$ The mechanical treatment should be considered a cornerstone of any plan of treatment. ${ }^{6}$

Our evaluation criteria were chosen to target specifically the effects of the protocols on pain and overall daily function (including work and/or recreation) as well as patient satisfaction. ${ }^{11}$ We also chose to evaluate measures with a questionnaire that generated feedback with regard to the patient's perceptions of the outcome, that is, the subject-relevant outcome measures which provide an additional measurement tool that concentrated on function and satisfaction and supplemented the pain subscale of the Foot Function Index. ${ }^{10}$

The pain subscale of the Foot Function Index was chosen because it is a validated instrument. ${ }^{12}$ Recent studies, including the multicenter clinical trial on acute plantar fasciitis by Pfeffer et al, ${ }^{13}$ have used the first seven items of the pain subscale of the Foot Function Index as the primary numeric outcome measure. In clinical practice, patients routinely complain about the severe pain with the first steps in the morning and focus on the pain when it is at its worst. Consequently, at the start of the study, we chose to independently analyze item 1 (worst pain) and item 2 (pain with first steps in the morning), since these were thought to be most clinically relevant to the patients' complaints. ${ }^{10}$ 
Table 2: Change Between Pain Subscale Scores of the Foot Function Index at Baseline and Eight-Week Follow-up Evaluation

\begin{tabular}{|c|c|c|c|c|c|c|c|}
\hline & \multicolumn{2}{|c|}{$\begin{array}{l}\text { plantar fascia } \\
\text { stretching exercise }\end{array}$} & \multicolumn{2}{|c|}{$\begin{array}{l}\text { TA stretching } \\
\text { exercise }\end{array}$} & \multicolumn{2}{|l|}{ Total } & \multirow[b]{2}{*}{ p-value } \\
\hline & Mean & Std. & Mean & Std. & Mean & Std. & \\
\hline & & Deviation & & Deviation & & Deviation & \\
\hline Item 1 baseline & 4.94 & .715 & 4.88 & .861 & 4.91 & .793 & 0.727 \\
\hline Itemlateightweek & 2.47 & .941 & 3.12 & .968 & 2.82 & 1.003 & 0.004 \\
\hline Difference of item1 & -2.50 & .861 & -1.71 & 1.006 & -2.10 & 1.012 & 0.002 \\
\hline Item 2 baseline & 4.58 & .732 & 4.83 & .824 & 4.72 & .788 & .164 \\
\hline Item2ateightweek & 2.33 & .862 & 3.10 & 1.100 & 2.74 & 1.062 & .001 \\
\hline Difference of item2 & -2.26 & .868 & -1.64 & 1.11 & -1.95 & 1.039 & 0.018 \\
\hline \multicolumn{8}{|l|}{ Combined scores } \\
\hline baseline & 25.69 & 3.319 & 26.12 & 3.710 & 25.92 & 3.519 & 0.599 \\
\hline \multicolumn{8}{|l|}{ Combined score } \\
\hline at eight week & 14.00 & 3.921 & 16.64 & 4.089 & 15.42 & 4.201 & 0.005 \\
\hline Difference of combined & -11.43 & 3.86 & -9.19 & 4.158 & -10.29 & 4.14 & 0.033 \\
\hline
\end{tabular}

In the study both groups reported an overall reduction in pain, the results of the analysis for the pain subscale scores of the Foot Function Index showed significant differences between the groups with respect to item 1 (worst pain; $p=0.002$ ), item 2 (first steps in the morning; $p=0.018)$, and all seven items were combined $(\mathrm{p}=0.033)$ with greater improvement noted in plantar fascia stretching group (Table 2).

In this study, analysis of the response rates to the outcome measures demonstrated a significant difference between the groups for all measures, with p values ranging between 0.016 and 0.049 (Table 3). The percentage of positive responses with regard to pain, activity limitations, and patient satisfaction was greater in plantar fascia stretching group than in TA stretching group. 
Table 3: Positive Response Rates by Group for Subject-Relevant Outcome Measures (SROM)

\begin{tabular}{|c|c|c|c|c|c|}
\hline Question (Definition of & Positive & Total & Positive & $95 \% \mathrm{CI}$ & p-value \\
\hline Positive Response) & responses & responses & responses $(\%)$ & & \\
\hline \multicolumn{6}{|l|}{ SROM1 $\uparrow$ (overall better off } \\
\hline \multicolumn{6}{|l|}{ than before treatment } \\
\hline \multicolumn{6}{|l|}{ plantar fascia stretching } \\
\hline exercise & 25 & 30 & 83.3 & 74.2 to 92.7 & 0.032 \\
\hline TA stretching exercise & 17 & 31 & 54.8 & 37.4 to 71.5 & \\
\hline \multicolumn{6}{|l|}{ SROM2(no heel pain or less } \\
\hline \multicolumn{6}{|l|}{ pain than before treatment) } \\
\hline \multicolumn{6}{|l|}{ plantar fascia stretching } \\
\hline exercise & 25 & 30 & 83.3 & 74.2 to 92.7 & 0.043 \\
\hline TA stretching exercise & 19 & 31 & 61.3 & 50.4 to 72.2 & \\
\hline \multicolumn{6}{|l|}{ SROM3(>50\% improvement } \\
\hline \multicolumn{6}{|l|}{ in heel pain) } \\
\hline plantar fascia stretching exercise & 24 & 30 & 80.0 & 69.3 to 90.6 & 0.036 \\
\hline TA stretching exercise & 10 & 31 & 32.3 & 21.5 to 43.4 & \\
\hline \multicolumn{6}{|l|}{ SROM4(heel pain all or } \\
\hline \multicolumn{6}{|l|}{ much better) } \\
\hline plantar fascia stretching exercise & 17 & 30 & 56.7 & 44.5 to 68.5 & $<0.001$ \\
\hline TA stretching exercise & 4 & 31 & 12.9 & 5.6 to 18.3 & \\
\hline \multicolumn{6}{|l|}{ SROM5(>50\% improvement in } \\
\hline \multicolumn{6}{|l|}{ work and/or recreational activities) } \\
\hline plantar fascia stretching exercise & 24 & 30 & 80.0 & 69.3 to 90.6 & 0.036 \\
\hline TA stretching exercise & 10 & 31 & 32.3 & 21.5 to 43.4 & \\
\hline \multicolumn{6}{|l|}{ SROM6(totally satisfied with } \\
\hline \multicolumn{6}{|l|}{ treatment or satisfied with minor } \\
\hline \multicolumn{6}{|l|}{ reservations) } \\
\hline plantar fascia stretching exercise & 26 & 30 & 86.7 & 77.3 to 95.7 & 0.036 \\
\hline TA stretching exercise & 10 & 31 & 32.3 & 21.5 to 43.4 & \\
\hline
\end{tabular}

Although improvement from the baseline symptoms was noted in both groups, the group managed with the non-weightbearing plantar fascia-stretching program was found to have superior results. These findings are encouraging, particularly considering that the patient population that was studied had plantar fasciitis for long duration and had exhausted numerous other treatment methods, including a standard Achilles tendon-stretching program. Our study showed that the Achilles tendon-stretching exercise had a beneficial effect, as illustrated by the improvement noted in the patients in TA stretching Group. However, it does not optimally isolate the plantar fascia and should be viewed as supplemental to the stretching exercise specific to the plantar fascia. Furthermore, the typical Achilles tendon-stretching exercise does 
not specifically recreate the windlass mechanism ${ }^{14}$ and it is routinely performed after the initiation of weight-bearing. We believe that it is important to commence stretching prior to weight bearing in the morning as weight-bearing without stretching may restart the cycle of microtearing and inflammation.

The plantar fascia-stretching protocol can be likened to the use of dorsiflexion night splints that incorporate toe dorsiflexion. ${ }^{15}$ In a recent prospective, randomized outcome study of patients with chronic plantar fasciitis, Powell et al. ${ }^{15}$ noted improvement of symptoms in $88 \%$ of the patients and satisfaction in $73 \%$ after use of a dorsiflexion night splint for one month. The plantar fascia-stretching protocol utilized in our study has two important advantages compared with use of a night splint. First, it eliminates the problem of poor compliance frequently associated with the use of bulky night splints. Second, the plantar fasciastretching exercises can be performed throughout the course of the day, especially prior to standing after prolonged sitting, which is not possible with the night splints. Lastly, the plantar fascia stretch may be more cost effective.

In addition to the prospective, randomized design, an additional strength of this study is the stringent method of patient selection. In order to minimize confounding variables, specific attention was paid to the inclusion of only patients who clearly had classic proximal plantar fasciitis. If they did not exhibit signs and symptoms of classic proximal plantar fasciitis, including tenderness localized to the medial tubercle of the calcaneus and pain with the first step in the morning, they were not enrolled in the study. A limitation of the study that could lead to a bias are that the duration of follow up was limited to eight weeks only and less number of patient .

\section{Conclusion}

Approximately $10 \%$ of patients with plantar fasciitis have development of persistent and often disabling symptoms. A program of non-weight-bearing stretching exercises specific to the plantar fascia is superior to the standard program of weight-bearing Achilles tendon-stretching exercises for the treatment of symptoms of proximal plantar fasciitis. These findings provide an alternative option to the present standard of care in the non-operative treatment of patients with chronic, disabling plantar fasciitis.

Conflict of interest: The authors declare that they have no conflict of interests.

\section{References}

1. Cornwall MW, McPoil TG. Plantar fasciitis: etiology and treatment. Journal of Orthopaedic \& Sports Physical Therapy 1999; 29:756-60.

2. Sammarco GJ, Helfrey RB. Surgical treatment of recalcitrant plantar fasciitis. Foot \& Ankle
International 1996; 17:520-6.

3. Schepsis AA, Leach RE, Gorzyca J. Plantar fasciitis. Etiology, treatment, surgical results, and review of the literature. Clin Orthop. 1991; 266:185-96.

4. Furey JG. Plantar fasciitis. The painful heel syndrome. J Bone Joint Surg Am. 1975; 57:672-3.

5. Steven K. Neufeld, MD Rebecca Cerrato,. MD.Plantar Fasciitis: Evaluatio and TreatmentJ Am Acad Orthop Surg 2008; 16:338-346

6. Steinmetz M. Treatment choices for plantar fasciitis. American Family Physician 1999; 60:2504

7. Mizel MS, Marymont JV, Trepman E. Treatment of plantar fasciitis with a night splint and shoe modification consisting of a steel shank and anterior rocker bottom. Foot \& Ankle International 1996;17:732-5.

8. Davis PF, Severud E, Baxter DE. Painful heel syndrome: results of non op-erative treatment. Foot Ankle Int. $1994 ; 15: 531-5$.

9. Martin RL, Irrgang JJ, Conti SF. Outcome study of subjects with insertion plantar fasciitis. Foot Ankle Int. 1998; 19:803-11.

10. Digiovanni BF, Nawoczenski DA, Lintal ME, et al. Tissue-specific plantar fascia-stretching exercise enhances outcomes in patients with chronic heel pain. A prospective, randomized study. J Bone Joint Surg Am. 2003; 85-A:1270-1277

11. Kitaoka HB, Alexander IJ, Adelaar RS, Nunley JA, Myerson MS, Sanders M. Clinical rating systems for the ankle-hindfoot, midfoot, hallux, and lesser toes. Foot Ankle Int. 1994;15:349-53.

12. Budiman-Mak E, Conrad KJ, Roach KE. The Foot Function Index: a measure of foot pain and disability. J Clin Epidemiol. 1991; 44:561-70.

13. Pfeffer G, Bacchetti P, Deland J. et al. Comparison of custom and prefabricated orthoses in the initial treatment of proximal plantar fasciitis. Foot \& Ankle International 1999; 20:214-21.

14. Hicks JH. The mechanics of the foot, II: the plantar aponeurosis and the arch. J Anat. 1954; 88:25-30.

15. Powell M, Post WR, Keener J, WeardenS. Effective treatment of chronic plantar fasciitis with dorsiflexion night splints:a crossover prospective randomized outcome study. Foot Ankle Int 1998; 19:10-8. 